Pacific Journal of Mathematics

ConstrectTING CoLoRE GRRP 


\title{
RECONSTRUCTING COLORED GRAPHS
}

\author{
JoSEPH M. WeINSTEIN
}

\begin{abstract}
A colored graph $G$ is a graph with given "coloring" (i.e., function) defined on the nodes and edges. A coloredgraph isomorphism is a graph isomorphism which preserves color values. $G$ is reconstructible if $G$ is isomorphic with $H$ whenever $H$ is a colored graph with the same nodes as $G$ and with $H-x$ isomorphic with $G-x$ for each node $x$. In this paper known reconstruction methods and results are generalized to colored graphs. A "colored" approach is used to give a simple proof of a recent "black-and-white" result: a block $G$ is reconstructible if $G$ has a node $z$ with $G-z$ a tree. New "colored" concepts and methods are used to deduce such results as the following: a colored graph $G$ is reconstructible if each color appears on at most three nodes.
\end{abstract}

Preliminaries. We write $|X|$ for the cardinality of a set $X$. By graph we mean henceforth colored graph, as above. Formally, a graph $G$ is the ordered triple $(N G, E G, C G)$, where $N G$ is the set of nodes, $E G$ is the set of edges, and $C G$ is the coloring. A graph $H$ is a subgraph of $G$ if $N H \subseteq N G, E H \subseteq E G$, and $C H \subseteq C G$ (i.e., $C H$ agrees with $C G$ ). A node (or edge) color class is the set of all nodes (all edges) of some color or set of colors. If the coloring is constant (on nodes, on edges), the graph is (node-, edge-)uncolored or (node-, edge-)monochrome.

Let $C, C^{\prime}$ be graph colorings for the same nodes and edges. $C^{\prime}$ is a refinement of $C$ if the values of $C^{\prime}$ determine those of $C$, i.e., if there is a function $f$ such that $C=f^{*} C^{\prime}$, where ${ }^{*}$ is functional composition (or, equivalently, if each $C$-class is a $C^{\prime}$-class). If this relation holds at least on nodes (or at least on edges), then $C^{\prime}$ is a node- (or an edge-)refinement of $C$. If $G, H$ are graphs with the same nodes and edges and with $\mathrm{CH}$ a (node-, edge-) refinement of $C G$, then $H$ is a (node-, edge-) refinement of $G$.

Let $G$ be a graph with $X$ a set of nodes and $x$ a node. $d x G$ is the degree of $x$ in $G$. $G / X$ is the restriction of $G$ to $X$, i.e., the largest subgraph of $G$ with nodes $X$ (or the subgraph of $G$ "induced" by $X) . \quad G-X$ is $G /(N G-X) ; G-x$ is $G-\{x\}$. The inventory of $G$ (over $X$ ) is the function which assigns to each isomorphism type $t$ of graphs the number of nodes $x$ (in $X$ ) such that $G-x$ is of type $t$. Recasting and generalizing the definition of the abstract, $G$ is reconstructible (over $X$ ) if its inventory (over $X$ ) determines its isomorphism type. (When $X$ is $N G$ we omit mention of $X$.) $G$ is $r r$ (refinably reconstructible) if every refinement of $G$ is reconstructible. 
Basic observations and techniques. For a moment let us consider "arbitrary graphs"-colored graphs with loops, multiple edges, and directed edges all allowed. The reconstruction problem for arbitrary graphs of given order $n$ is readily seen to be equivalent to the same problem for just the uncolored graphs of order $n$, or for just the simple graphs of order $n$ (where "simple" means "without loops or multiple edges"). Indeed, an arbitrary graph $G$ can be "represented" as an uncolored graph $H$ with the same nodes or as a simple graph $H^{\prime}$ with the same nodes, for we can "code" the node colors and loop color patterns either in the form of multiple loops (in $H$ ) or in the form of node colors (in $H^{\prime}$ ); and similarly for the edge colors and edge color patterns. Henceforth, all graphs discussed in this paper are finite, simple, and undirected. An edge between nodes $x$ and $y$ will be identified with the pair-set $\{x, y\}$. We shall usually abbreviate "CG(\{x,y\})" to " $C G(x, y)$ ".

To reconstruct all graphs it suffices to reconstruct all complete graphs, for every graph may be regarded as a complete graph with one extra color for the originally missing edges. Further, while for convenience we consider arbitrary node coloration, it would usually suffice to consider just node-monochrome graphs, i.e., graphs in which we color only the edges. The following lemma justifies this statement.

LEMMA 1. Every graph $G$ of order $\geqq 3$ has a node-monochrome edge-refinement $H$ such that if $H$ is reconstructible then so is $G$, except possibly when $G$ is both connected and bipartite.

Proof. If $G$ is not connected, $G$ is reconstructible (without reference to any $H$ : see remarks after 3 below), so we assume that $G$ is connected. To each edge $\{x, y\}$ of $G$ we assign the $H$-color $(\{C G(x)$, $C G(y)\}, C G(x, y))$. Note that the inventory of $G$ determines that of $H$. It is clear that from reconstruction of $H$ we obtain the edge colors of $G$; there remains to show that we can recover the node colors as well. To do this, for each node $x$ let $M x$ be the set of all $G$-colors $c$ such that $c$ belongs to the first coordinate of the $H$-color of every edge incident to $x$, and let $m x$ be $|M x|$. If $m x=1$ we recover the $G$-color of $x$ as the unique member of $M x$. If $m x=2$ and $x$ adjoins a node $z$ for which we have "already" recovered the $G$-color, we may recover the $G$-color of $x$ by examining the first coordinate of the $H$-color of $\{x, z\}$. Thus (formally by using induction on the distance of $x$ from the nearest node $z$ with $m z=1$ ) we find that the only case in which possibly the G-color of a node $x$ cannot be recovered is when every node satisfies $m=2$. This means that the node coloring of $G$ is proper (i.e., adjoining nodes receive 
distinct colors) and (because $G$ is connected) there can be just 2 colors, so $G$ is bipartite.

Proposition 2. All graphs of orders $3,4,5$ are reconstructible.

Proof. By the preceding remarks and lemma, it suffices to consider complete node-monochrome graphs; by "brute force" one can then go case-by-case according to the numbers of edges in each color class and then sometimes by subcases based on the arrangements of these edges.

In generalizing the reconstruction problem from uncolored to colored graphs, it is reassuring that the basic known tool for this problem generalizes too.

Lemma 3. (Kelly [1].) For any graph $G$ and for any graph $K$ of order less than the order of $G$ and for any node $x$ of $G$ : the inventory of $G$ determines the nnmber of subgraphs of $G$ isomorphic with $K$, and this inventory and the isomorphism type of $G-x$ together determine the number of subgraphs of $G$ isomorphic with $K$ and containing $x$.

Known uncolored reconstruction results which depend essentially only on Kelly's lemma extend readily to (colored) graphs. In particular, a graph of order 3 or more is reconstructible if it is not connected, or it is a tree, or if it has a cut node but no end node. There is also the following little-noted but useful consequence.

CoRollary 4. For any graph $G$ and node $x$ of $G$ and integer $k$, the inventory of $G$ and the isomorphism type of $G-x$ together determine both $d x G$ and the number of nodes $y$ adjoining $x$ in $G$ with $d y G=k$.

New techniques. Our main new idea is the concept $r r$ (refinably reconstructible). This concept has no ready analog in black-and-white, and in a sense it permits argument which reverses the direction of attack customary in the uncolored case, as follows. Every uncolored graph may be regarded as a complete graph with at most two edge colors, the easy subcase being when there is only one color(!). But with $r r$ two colors may be too few rather than too many for easy solution. The use of $r r$ allows induction on the order: we can sometimes reduce the problem of a large graph being $r r$ to the problem of a smaller graph being $r r$. The following simple lemma and corollary illustrate this idea.

Lemma 5. Suppose $X$ is a node color class such that $G / X$ is rigid 
(i.e., has only one automorphism). Then $G-X$ has a refinement $H$ such that if $H$ is reconstructible then so is $G$ (and then $G$ is reconstructible over $N G-X$ ).

Proof. We wish to define $H$ so that the inventory of $G$ (over $N G-X$ ) determines the inventory of $H$ and (at the same time) so that we can recover all needed information about $G$ from a reconstruction of $H$. It is easily checked that it suffices to define $H$ as follows. For each node $u$ of $G-X$ and each edge $\{u, v\}$ of $G-X$ let $C H(u)$ be the isomorphism type of the graph $G /(X \cup\{u\})$ and let $C H(u, v)$ be the isomorphism type of the graph $G /(X \cup\{u\} \cup\{v\})$.

Corollary. Suppose $X$ is a node color class with $G / X$ rigid and $G-X r r$. Then $G$ is $r r$.

Proof. In any refinement $G^{\prime}$ of $G, X$ will be a color class with $G^{\prime} / X$ rigid. Apply the lemma to $G^{\prime}$ and $X$ : since $G-X$ is $r r, H$ will be reconstructible, so $G^{\prime}$ will be reconstructible.

Let us now give an example of the way in which simple "color" reductions such as this corollary can, when combined with simple "color" generalizations of "uncolored" theorems, sometimes yield notso-simple "uncolored" consequences.

Proposition 6. Suppose $G$ is a graph of order at least 4 and $z$ $a$ node of $G$ such that $G-z$ is a tree and $d z G$ exceeds $d x G+1$ for all nodes $x \neq z$. Then $G$ is $r r$.

Proof. Thanks to the degree condition we can "paint" $z$ a special color which is "recognizable" in every subgraph $G-x$. By the Corollary of 5, with $X=\{z\}$, and by the fact that trees are $r r, G$ is $r r$.

The following result was announced for the uncolored case by $\mathrm{O}^{\prime} \mathrm{Neill}$ in [3]. Given a graph $G$ call a node $x$ constant if all edges incident with $x$ have the same color (and then write $c x$ for this color).

Theorem 7. Suppose $G$ is a block with node $z$ such that $G-z$ is a tree with $h$ end nodes, where: if $h$ is at most 3 then $G$ is monochrome, and if $h$ exceeds 3 then each node of degree at least 3 is constant. Then $G$ is reconstructible.

Proof. Choose such $z$ and let $H$ be $G-z$. $G$ is a block, so $z$ adjoins each of the $h$ end nodes of $H$. On the other hand, each node of the tree $H$ has degree in $H$ at most $h$ : it follows that $z$ has maximum degree in $G$. However, we may assume that at least one 
node $x \neq z$ has degree in $G$ at least $d z G-1$ : else, by $6, G$ is already reconstructible. Since such $x$ has degree in $G$ at most $h+1, d z G$ is at most $h+2$, and $d z G=h+2$ only if $d x H=h$ and $x$ adjoins $z$.

We may assume that $d z G$ exceeds $h$, else we reconstruct $G$ from $H$ by putting in the edges (all of color $c z$ ) from $z$ to the end nodes of $H$. Hence $d z G=h+1$ or $h+2$. When $h=2$ special argument now determines the isomorphism type of $G$, so we assume that $h$ is at least 3. Now fix $x$ to be any node with degree in $G$ at least $d z G-1$. Note that either $d x H=h$ and then $x$ is the only node in $H$ of degree 3 or more in $H$, or else $d x H=h-1$ and then (besides $x) H$ has exactly one node $u$ of degree more than 2 in $H$, and indeed $d u H=3$.

If $d x G=d z G$, then $d z G=h+1$, and $x$ adjoins $z$, and we reconstruct $G$ from $H$ by drawing edges from $z$ to each end node of $H$ and to the unique node of degree $h$ in $H$. So we assume that $d x G=$ $d z G-1$.

Suppose $d z G=h+2$ with $h$ at least 4. In $H, x$ has degree $h$ and every other node has degree at most 2 ; and $z$ adjoins exactly one node $y$ of $H$ other than $x$ and the end nodes. In $G, y$ is the unique node of degree 3 . By 4, we can determine whether $y$ adjoins $x$ and we can determine the isomorphism types of $G-y, G-x$. If $y$ adjoins $x$ we reconstruct $G$ from $G-y$ by drawing edges (of color $c y$ ) from $y$ to the unique nodes in $G-y$ of degrees $1, h, h+1$. If $y$ does not adjoin $x$, we reconstruct $G$ from $G-x$ by drawing edges (of color $c x$ ) to the nodes of degrees $h+1$ and 1 in $G-x$.

Suppose $d z G=h+1$. If $h$ exceeds 4 and some node of degree $h$ in $G$ adjoins $z$ (which, by 4 , we can check) then $G$ is reconstructed from $H$ by joining $z$ to the end nodes of $H$ and to the unique node of degree $h-1$ in $H$. If $h$ is 4 or more and some node $x$ of degree $h$ in $G$ does not adjoin $z, z$ adjoins exactly one node $y(\neq x)$ of $H$ other than the end nodes, and (in $G$ ) $y$ is the unique node of degree 3. Analogously with the preceding paragraph, if $y$ adjoins $x$ we reconstruct $G$ by drawing edges from $y$ to the nodes of degrees $1, h-1, h$ in $G-y$; and if $y$ does not adjoin $x$ we draw edges from $x$ to the nodes of degree 1 in $G-x$.

Just three cases are not covered by the last two paragraphs:

(1) $h=4, d z G=5$, and $z$ adjoins some node $x$ of degree 4 in $G$.

(2) $h=3, d z G=5$, and $z$ adjoins nodes $x, y$ of degrees 4,3 in $G$.

(3) $h=3, d z G=4$, and $z$ adjoins a node $y$ of degree 3 in $G$ but not the other node $x$ of degree 3 in $G$.

In case (1) $G$ has a unique node $u$ of degree 3 and $u$ does not adjoin $z$. We reconstruct $G$ from $G-u$ by adding edges from $u$ to nodes of degree 1 and (if such exists) of degree 3 in $G-u$. In case 
(2) reconstruct $G$ from $G-x$ by drawing an edge from $x$ to each node of degree 1 or 4 in $G-x$ and - if $G-x$ has no node of degree 3 - draw one edge from $x$ to some node adjoining the node of degree 4 in $G-x$ and on a (in fact the unique) circuit in $G-x$. (This part of our argument uses the assumed monochromicity for $h=3$.) In case (3) reconstruct $G$ similarly from $G-x$ but without drawing the edge to the node of degree 4 in $G-x$.

Further reduction. Lemma 5 and its corollary reduced the problem of a graph $G$ being $r r$ to the same problem for a smaller graph $G-X$, at least when $X$ is a color class with $G / X$ rigid. We now show that such reduction is possible under weaker hypotheses on $G / X$, at least when (as 1 shows we may as well suppose) $G$ is complete.

THEOREM 8. Suppose $G$ is complete and $X$ a node color class such that $G / X$ has at most two automorphisms. Then $G-X$ has a refinement $H$ such that if $H$ is reconstructible then so is $G$ (and then $G$ is reconstructible over $N G-X$ ).

Corollary. With $G, X$ as in the hypotheses of the theorem, if $G-X$ is $r r$ so is $G$. (The proof is analogous to the proof from Lemma 5 of its corollary.)

Proof of the theorem. Define $H$ as in the proof of 5. Clearly, the inventory of $G$ (over $N G-X$ ) determines the inventory of $H$. We must show that when $H$ is reconstructed so is $G$. That is, let $G^{\prime}$ be a graph with the same nodes as $G$ and with $G^{\prime}-x$ isomorphic with $G-x$ for all nodes $x$ and with $H^{\prime}$ constructed from $G^{\prime}$ by the procedure of the proof of 5. Suppose $f$ an isomorphism from $H$ to $H^{\prime}$. It is easily seen that $f$ is an isomorphism from $G-X$ to $G^{\prime}-X$ : we would like to show how to extend $f$ to an isomorphism from $G$ to $G^{\prime}$.

Let us say that a 1-1 map $g$ on $X$ onto $X$ works at a given node $u$ of $G-X$ if $g \cup\{(u, f(u))\}$ is an isomorphism of $G /(X \cup\{u\})$ with $G^{\prime} /(X \cup\{f(u)\})$. The desired extension of $f$ will be of the form $f \cup g$, where $g$ is a $1-1$ map on $X$ onto $X$ which works at each node $u$ of $N G-X$. We must show how to define such $g$.

In case $N G-X$ has a node $u$ with $G /(X \cup\{u\})$ rigid, only one $g$ is possible; that $g$ works at each $v \in N G-X-\{u\}$ is guaranteed by the definition of $C H(u, v)$ and by the fact that $f$ preserves the $H$-color of $\{u, v\}$. If no such $u$ exists, $g$ may be taken any isomorphism (of the at most two) of $G / X$ with $G^{\prime} / X$.

Theorem 9. Suppose $G$ is complete and $X$ a node color class 
with $|X|$ at most 3. Then $G-X$ has a refinement $H$ such that if $H$ is reconstructible then so is $G$.

Corollary. With $G, X$ as in the hypotheses of the theorem, if $G-X$ is $r r$ so is $G$.

Proof of the theorem. Again, define $H$ as in the proof of 5 . As in the proof of 8 , our problem is again defining a $g$ which works for all $u \in N G-X$.

We may assume that $|X|=3$ and that $G / X$ is both node- and edge- monochrome: else the hypotheses of 8 hold and we are done. Thus, in order that $g$ works at $u$ it suffices that $g$ "preserve edge color from $u$ to $X$ ", i.e., for each $x \in X C G(u, x)=C G^{\prime}(f(u), g(x))$. We shall call $u$ constant if only one color appears on the edges from $u$ to $X$. More generally, we say that a color appears ( $k$ times) at $u$ if it is the $G$-color of some (exactly $k$ ) edges from $u$ to $X$.

We may assume that for each set $T$ of precisely three colors it is not the case that every node $u$ of $G-X$ is either constant or has precisely the colors of $T$ appearing. For in such case, fix $x \in X$. We reconstruct $G$ directly (without use of $H$ or of $f$ ) by coloring each edge $\{u, x\}(u \in N G-X)$ as follows: if the two other edges from $u$ to $X$ have the same color, give $\{u, x\}$ this color; if not, give $\{u, x\}$ the color of $T$ missing from these edges.

Case (1). At some node u of $G-X 3$ colors appear. Call these colors $1,2,3$. Then $g$ is determined by $C H(u)$ and by the requirement that $g$ works at $u$. We must show that $g$ works at each node $v$ of $N G-X-\{u\}$. Fix $v$ and let $P$ be the set of all ordered pairs $(i, j)$ such that for some $x \in X i$ is the $G$-color of $\{u, x\}$ and $j$ is the $G$-color of $\{v, x\}$. The definition of $C H(u, v)$ and the fact that $f$ preserves this $H$-color ensure that $g$ does work at $v$, except possibly when $P$ is a cyclic permutation of $\{1,2,3\}$, which we now assume is the case. By the previous paragraph, $G-X$ has a node $w$ which is not constant and at which not all the colors 1, 2,3 appear. Some color $k$ appears exactly once at $w$. Using this fact and using the values of $C H(u, w)$ and of $C H(v, w)$ and the fact that $P$ is a cyclic permutation of $\{1,2,3\}$ one may check that $g$ does work at $v$.

Case (2). At no node do 3 colors appear. Hence at every nonconstant node $u$ exactly one color appears once and exactly one color appears twice. Let $Q$ be the set of all ordered pairs $\left(x, x^{\prime}\right)$ with $x, x^{\prime}$ in $X$ and such that for at least one node $u$ of $G-X$ the color on $\{u, x\}$ appears just once at $u$ and is the color on $\left\{f(u), x^{\prime}\right\}$ in $G^{\prime}$. The definition of $\mathrm{CH}$ and the fact that $f$ preserves $\mathrm{CH}$-edge-colors 
ensure that $Q$ is a 1-1 function. Let $g$ be any 1-1 extension of $Q$ on $X$ onto $X$.

Note and example. In the conclusion of Theorem 9 we cannot assert (in analogy with 5 or 8) that $G$ is reconstructible over $N G-X$. The argument in the proof of 9 in the paragraph before case (1) uses the inventory of $G$ over $\{x\} \cup(N G-X)$ with $x \in X$. The following example shows this use essential. Let $G$ be the complete graph on the nodes $0,1,2,3,4,5$ with $X=\{3,4,5\}$ and with the following coloration. $C G(x)=0$ for $x \notin X$ and 1 for $x \in X$; $C G(x, y)=3$ if $x, y$ both $\in X ; C G(x, y)=x+y(\bmod 3)$ (=remainder on division of $x+y$ by 3$)$ for $x \in X$ and $y \notin X ; C G(0,1)=0, C G(1,2)=1, C G(0,2)=2$. $G$ is not reconstructible over $N G-X$; for let $G^{\prime}$ be the same as $G$ except that $C G^{\prime}(1,2)=2, C G^{\prime}(0,2)=1 . G$ and $G^{\prime}$ are not isomorphic, yet they have the same inventory over $N G-X\left(=N G^{\prime}-X\right)$ and $G-X, G^{\prime}-X$ are both $r r$.

THEOREM 10. Let $G$ be a graph whose nodes can be partitioned into node color classes $X$ such that one $X$ satisfies $|X|=3,4$, or 5 and each remaining $X$ satisfies either: $|X|=3$ or $G / X$ has at most two automorphisms. Then $G$ is $r$.

Proof. By induction on the number of classes $X$, using the Corollaries of 8 and 9 along with 2.

\section{REFERENCES}

1. P. Kelly, A congruence theorem for trees, Pacific J. Math., 7 (1957), 961-968.

2. B. Manvel, A bibliography on reconstruction of graphs, distributed at annual meeting of Amer. Math. Soc., Jan. 1974, San Francisco.

3. P. O'Neill, Reconstruction of a class of blocks, abstract in Notices Amer. Math. Soc., Jan. 21, 1974, A39.

4. J. Weinstein, Reconstructing colored graphs, abstract in Notices Amer. Math. Soc., Jan. 21, 1974, A34. [Same title used for talk at annual meeting of Amer. Math. Soc., Jan. 1974, San Francisco and for preprint distributed at the talk.]

Received June 4, 1974.

St. Mary's College of Maryland 


\title{
PACIFIC JOURNAL OF MATHEMATICS
}

\author{
EDITORS
}

RICHARD ARENS (Managing Editor)

University of California

Los Angeles, California 90024

R. A. Beaumont

University of Washington

Seattle, Washington 98105
J. DugundJI

Department of Mathematics University of Southern California Los Angeles, California 90007

D. Gilbarg and J. Milgram

Stanford University

Stanford, California 94305

\section{ASSOCIATE EDITORS}

E. F. BECKENBACH

B. H. NeUManN

F. WOLF

K. YosHIDA

\section{SUPPORTING INSTITUTIONS}

UNIVERSITY OF BRITISH COLUMBIA

GALIFORNIA INSTITUTE OF TECHNOLOGY

UNIVERSITY OF CALIFORNIA

MONTANA STATE UNIVERSITY

UNIVERSITY OF NEVADA

NEW MEXICO STATE UNIVERSITY

OREGON STATE UNIVERSITY

UNIVERSITY OF OREGON

OSAKA UNIVERSITY
UNIVERSITY OF SOUTHERN CALIFORNIA

STANFORD UNIVERSITY

UNIVERSITY OF TOKYO

UNIVERSITY OF UTAH

WASHINGTON STATE UNIVERSITY

UNIVERSITY OF WASHINGTON

AMERICAN MATHEMATICAL SOCIETY NAVAL WEAPONS CENTER 


\section{Pacific Journal of Mathematics}

\section{Vol. 57, No. $1 \quad$ January, 1975}

Keith Roy Allen, Dendritic compactification ......................... 1

Daniel D. Anderson, The Krull intersection theorem ..................... 11

George Phillip Barker and David Hilding Carlson, Cones of diagonally dominant

matrices .............................................

David Wilmot Barnette, Generalized combinatorial cells and facet splitting ........

Stefan Bergman, Bounds for distortion in pseudoconformal mappings ............

Nguyên Phuong Các, On bounded solutions of a strongly nonlinear elliptic

equation ...............................................

Philip Throop Church and James Timourian, Maps with 0-dimensional critical

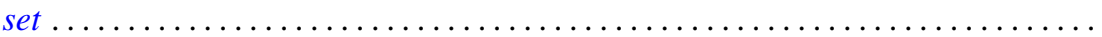

G. Coquet and J. C. Dupin, Sur les convexes ubiquitaires ................. 67

Kandiah Dayanithy, On perturbation of differential operators............... 85

Thomas P. Dence, A Lebesgue decomposition for vector valued additive set

functions .............................................. 91

John Riley Durbin, On locally compact wreath products.................. 99

Allan L. Edelson, The converse to a theorem of Conner and Floyd ............. 109

William Alan Feldman and James Franklin Porter, Compact convergence and the

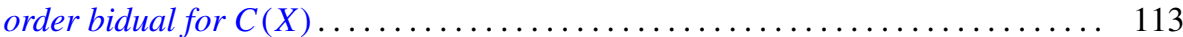

Ralph S. Freese, Ideal lattices of lattices......................... 125

R. Gow, Groups whose irreducible character degrees are ordered by divisibility ... 135

David G. Green, The lattice of congruences on an inverse semigroup ............ 141

John William Green, Completion and semicompletion of Moore spaces .......... 153

David James Hallenbeck, Convex hulls and extreme points of families of starlike and close-to-convex mappings .................................. 167

Israel (Yitzchak) Nathan Herstein, On a theorem of Brauer-Cartan-Hua type ...... 177

Virgil Dwight House, Jr., Countable products of generalized countably compact spaces ............................................ 183

John Sollion Hsia, Spinor norms of local integral rotations. I . . . . . . ......... 199

Hugo Junghenn, Almost periodic compactifications of transformation

semigroups....................................

Shin'ichi Kinoshita, On elementary ideals of projective planes in the 4-sphere and oriented $\Theta$-curves in the 3 -sphere ............................ 217

Ronald Fred Levy, Showering spaces............................ 223

Geoffrey Mason, Two theorems on groups of characteristic 2-type............. 233

Cyril Nasim, An inversion formula for Hankel transform ................... 255

W. P. Novinger, Real parts of uniform algebras on the circle ................ 259

T. Parthasarathy and T. E. S. Raghavan, Equilibria of continuous two-person

games............................................... 265

John Pfaltzgraff and Ted Joe Suffridge, Close-to-starlike holomorphic functions of several variables ....................................... 271

Esther Portnoy, Developable surfaces in hyperbolic space .................. 281

Maxwell Alexander Rosenlicht, Differential extension fields of exponential type . . . 289

Keith William Schrader and James Lewis Thornburg, Sufficient conditions for the

existence of convergent subsequences ........................... 301

Joseph M. Weinstein, Reconstructing colored graphs .................. 307 